Hardy-Ramanujan Journal

Vol. 33 (2010) pp. 32-58

\title{
ON THE MELLIN TRANSFORMS OF POWERS OF HARDY'S FUNCTION
}

\author{
Aleksandar IVIĆ
}

Abstract. Various properties of the Mellin transform function

$$
\mathcal{M}_{k}(s):=\int_{1}^{\infty} Z^{k}(x) x^{-s} \mathrm{~d} x
$$

are investigated, where

$$
Z(t):=\zeta\left(\frac{1}{2}+i t\right) \chi\left(\frac{1}{2}+i t\right)^{-1 / 2}, \quad \zeta(s)=\chi(s) \zeta(1-s)
$$

is Hardy's function. Connections with power moments of $\left|\zeta\left(\frac{1}{2}+i t\right)\right|$ are established, and natural boundaries of $\mathcal{M}_{k}(s)$ are discussed.

\section{INTRODUCTION}

Power moments of $\left|\zeta\left(\frac{1}{2}+i t\right)\right|$ are a central problem in the theory of the Riemann zeta-function

$$
\zeta(s)=\sum_{n=1}^{\infty} n^{-s} \quad(\sigma=\Re \mathrm{e} s>1),
$$

which admits analytic continuation to $\mathbb{C}$, having only a simple pole at $s=1$. A vast literature exists on this subject (see e.g., the monographs [10], [11], [26] and $[28]$ ). One way to tackle them is to deal with the (modified) Mellin transform function

$$
\mathcal{Z}_{k}(s):=\int_{1}^{\infty}\left|\zeta\left(\frac{1}{2}+i x\right)\right|^{2 k} x^{-s} \mathrm{~d} x \quad(k \in \mathbb{N})
$$

1991 Mathematics Subject Classification. 11 M 06.

Key words and phrases. Hardy's function, Mellin transforms, power moments, Riemann zetafunction,. 
where $\sigma=\Re$ e $s$ is so large that the integral in (1.1) converges absolutely. These functions in the cases when $k=1$ or $k=2$ have been intensively investigated (e.g., see the works [14], [18], [23] and [24]). It is known that $\mathcal{Z}_{1}(s)$ has meromorphic continuation to $\mathbb{C}$. It has a pole of order two at $s=1$ and the principal part of the Laurent expansion at $s=1$ of $\mathcal{Z}_{1}(s)$ is

$$
\frac{1}{(s-1)^{2}}+\frac{2 \gamma-\log (2 \pi)}{s-1}
$$

where $\gamma=-\Gamma^{\prime}(1)=0.577215 \ldots$ is Euler's constant. It also has simple poles at $s=-1,-3, \ldots$, whose residues can be expressed explicitly in term of Bernoulli numbers (see M. Lukkarinen [23]).

The analytic continuation of $\mathcal{Z}_{2}(s)$ has also (see e.g., Y. Motohashi [24]) infinitely many poles. Namely in the half-plane $\Re$ e $s>0$ it has the following singularities: the pole $s=1$ of order five, simple poles at $s=\frac{1}{2} \pm i \kappa_{j}\left(\kappa_{j}=\sqrt{\lambda_{j}-\frac{1}{4}}\right)$ and poles at $s=\rho / 2$. Here $\rho$ denotes complex zeros of $\zeta(s)$, and $\left\{\lambda_{j}=\kappa_{j}^{2}+\frac{1}{4}\right\} \cup$ $\{0\}$ is the discrete spectrum of the non-Euclidean Laplacian acting on $S L(2, \mathbb{Z})$ automorphic forms. This shows that $\mathcal{Z}_{2}(s)$ has a different and more complicated structure than $\mathcal{Z}_{1}(s)$.

Instead of $\mathcal{Z}_{k}(s)$ one can consider the more general Mellin transform function

$$
\mathcal{M}_{k}(s):=\int_{1}^{\infty} Z^{k}(x) x^{-s} \mathrm{~d} x \quad(k \in \mathbb{N})
$$

where again $\sigma=\Re e s$ is so large that the integral in (1.2) converges absolutely. Here $Z(x)$ is the classical Hardy function, defined as

$$
Z(t):=\zeta\left(\frac{1}{2}+i t\right)\left(\chi\left(\frac{1}{2}+i t\right)\right)^{-1 / 2}, \quad \zeta(s)=\chi(s) \zeta(1-s)
$$

with

$$
\chi(s)=2^{s} \pi^{s-1} \sin \left(\frac{1}{2} \pi s\right) \Gamma(1-s), \quad \chi(s) \chi(1-s)=1 .
$$

It follows that $\overline{\chi\left(\frac{1}{2}+i t\right)}=\chi\left(\frac{1}{2}-i t\right)$, so that $Z(t) \in \mathbb{R}$ when $t \in \mathbb{R}$ and $|Z(t)|=$ $\left|\zeta\left(\frac{1}{2}+i t\right)\right|$. Thus the zeros of $\zeta(s)$ on the "critical line" $\Re$ e $s=1 / 2$ correspond to the real zeros of $Z(t)$, which makes $Z(t)$ an invaluable tool in the study of the zeros of the zeta-function on the critical line. Note that when $k=2 \ell$ is even, then

$$
\mathcal{M}_{2 \ell}(s)=\int_{1}^{\infty} Z^{2 \ell}(x) x^{-s} \mathrm{~d} x=\int_{1}^{\infty}\left|\zeta\left(\frac{1}{2}+i x\right)\right|^{2 \ell} x^{-s} \mathrm{~d} x=\mathcal{Z}_{\ell}(s)
$$

in former notation. Hence $\mathcal{M}_{k}(s)$ is also closely connected to the moments of $\zeta\left(\frac{1}{2}+i x\right)$. If we define, for fixed $k \in \mathbb{N}$, the $k$-th moment of Hardy's function as

$$
\mathcal{I}_{k}(x):=\int_{1}^{x} Z^{k}(y) \mathrm{d} y,
$$


then on integrating by parts we find that

$$
\mathcal{M}_{k}(s)=s \int_{1}^{\infty} \mathcal{I}_{k}(x) x^{-s-1} \mathrm{~d} x
$$

so that the properties of $\mathcal{I}_{k}(x)$ are reflected on $\mathcal{M}_{k}(s)$. Conversely, the Mellin inversion formula gives

$$
Z^{k}(x)=\frac{1}{2 \pi i} \int_{(c)} \mathcal{M}_{k}(s) x^{s-1} \mathrm{~d} s
$$

for suitable $c(>0)$. From (1.6) we obtain by integration

$$
\mathcal{I}_{k}(x)=\frac{1}{2 \pi i} \int_{(c)} \mathcal{M}_{k}(s) \frac{x^{s}}{s} \mathrm{~d} s+O(1)
$$

The plan of the paper is as follows. In Section 2 we consider $\mathcal{M}_{k}(s)$ and obtain some general results for this modified Mellin transform. Section 3 is devoted to $\mathcal{M}_{k}(s)$ in the special cases when $k=1$ and $k=3$. The discussion related to the analytic continuation of $\mathcal{M}_{3}(s)$ is made by the use of the cubic moment of $Z(x)$, which is dealt with in Section 4. Finally the natural boundaries of $\mathcal{M}_{k}(s)$ and related problems are treated in Section 5.

\section{Properties of $\mathcal{M}_{k}(s)$}

First we recall that the Mellin transform of $f(x)$ is commonly defined as

$$
\mathcal{M}[f(x)]=F(s):=\int_{0}^{\infty} f(x) x^{s-1} \mathrm{~d} x \quad(s=\sigma+i t) .
$$

Mellin and Laplace transforms play an important rôle in Analytic Number Theory. They can be viewed, by a change of variable, as special cases of Fourier transforms, and their properties can be deduced from the general theory of Fourier transforms (see e.g., E.C. Titchmarsh [27]). For example, by the change of variable $x=$ $\mathrm{e}^{t}, z=s-1,(1.2)$ becomes

$$
\int_{0}^{\infty} Z^{k}\left(\mathrm{e}^{t}\right) \mathrm{e}^{-z t} \mathrm{~d} t \quad(\Re \mathrm{e} z>0)
$$

which is the Laplace transform of $Z^{k}\left(\mathrm{e}^{t}\right)$. The reason that we have defined in (1.1) and (1.2) somewhat differently the Mellin transforms $\mathcal{Z}_{k}(s), \mathcal{M}_{k}(s)$ is practical: the lower limit of integration $x=1$ dispenses with potential convergence problems at $x=0$, while the appearance of $x^{-s}$ instead of the familiar $x^{s-1}$ stresses the analogy with Dirichlet series where one has a sum of $f(n) n^{-s}$ and not $f(n) n^{s-1}$. 
One of the basic properties of Mellin transforms is the inversion formula

$$
\frac{1}{2}\{f(x+0)+f(x-0)\}=\frac{1}{2 \pi i} \int_{(\sigma)} F(s) x^{-s} \mathrm{~d} s=\frac{1}{2 \pi i} \lim _{T \rightarrow \infty} \int_{\sigma-i T}^{\sigma+i T} F(s) x^{-s} \mathrm{~d} s .
$$

Formula (2.2) certainly holds if $f(x) x^{\sigma-1} \in L(0, \infty)$, and $f(x)$ is of bounded variation on every finite $x$-interval. Therefore the inversion formula (1.6) follows from (2.2) by a change of variable. Note that if $G(s)$ denotes the Mellin transform of $g(x)$ then, assuming $f(x)$ and $g(x)$ to be real-valued, we formally have

$$
\begin{aligned}
& \frac{1}{2 \pi i} \int_{(\sigma)} F(s) \overline{G(s)} \mathrm{d} s=\int_{0}^{\infty} g(x)\left(\frac{1}{2 \pi i} \int_{(\sigma)} F(s) x^{\sigma-i t-1} \mathrm{~d} s\right) \mathrm{d} x \\
& =\int_{0}^{\infty} g(x) x^{2 \sigma-1}\left(\frac{1}{2 \pi i} \int_{(\sigma)} F(s) x^{-s} \mathrm{~d} s\right) \mathrm{d} x=\int_{0}^{\infty} f(x) g(x) x^{2 \sigma-1} \mathrm{~d} x .
\end{aligned}
$$

The relation (2.3) is a form of Parseval's formula for Mellin transforms, and it offers various possibilities for mean square bounds. A condition under which (2.3) holds is that $x^{\sigma} f(x)$ and $x^{\sigma} g(x)$ belong to $L^{2}((0, \infty), \mathrm{d} x / x)$. A variant of $(2.3)$ is (see [27, Theorem 73])

$$
\frac{1}{2 \pi i} \int_{(c)} F(w) G(s-w) \mathrm{d} w=\int_{0}^{\infty} f(x) g(x) x^{s-1} \mathrm{~d} x,
$$

which holds if $x^{c} f(x)$ and $x^{\sigma-c} g(x)$ belong to $L^{2}((0, \infty), \mathrm{d} x / x)$, where as usual

$$
L^{p}(a, b):=\left\{\left.f(x)\left|\int_{a}^{b}\right| f(x)\right|^{p} \mathrm{~d} x<\infty\right\} .
$$

Our first result is

THEOREM 1. For $c \geqslant c_{k}>0, k \geqslant 2$ and $\sigma=\Re$ e $s \geqslant \sigma_{1}(k)(>1)$ we have

$$
\mathcal{M}_{k}(s)=\frac{1}{2 \pi i} \int_{(c)} \mathcal{M}_{k-r}(w) \mathcal{M}_{r}(1-w+s) \mathrm{d} w \quad(r=1, \ldots, k-1) .
$$

In particular, for $\sigma>c>1$,

$$
\mathcal{M}_{3}(s)=\frac{1}{2 \pi i} \int_{(c)} \mathcal{M}_{1}(w) \mathcal{M}_{2}(1-w+s) \mathrm{d} w .
$$

Proof. Consider

$$
f(x)=Z^{k-r}\left(\frac{1}{x}\right) \frac{1}{x}, \quad g(x)=Z^{r}\left(\frac{1}{x}\right) \frac{1}{x} \quad(0<x \leqslant 1),
$$


and $f(x)=g(x)=0$ if $x>1$. With the change of variable $y=1 / x$ we have

$$
F(s)=\int_{0}^{\infty} f(x) x^{s-1} \mathrm{~d} x=\int_{0}^{1} Z^{k-r}\left(\frac{1}{x}\right) x^{s-2} \mathrm{~d} x=\int_{1}^{\infty} Z^{k-r}(y) y^{-s} \mathrm{~d} y=\mathcal{M}_{k-r}(s),
$$

and likewise $G(s)=\mathcal{M}_{r}(s)$. Hence (2.4) yields

$$
\frac{1}{2 \pi i} \int_{(c)} \mathcal{M}_{k-r}(w) \mathcal{M}_{r}(s-w) \mathrm{d} w=\int_{0}^{1} Z^{k-r}\left(\frac{1}{x}\right) Z^{r}\left(\frac{1}{x}\right) x^{s-3} \mathrm{~d} x=\mathcal{M}_{k}(s-1),
$$

again with the change of variable $y=1 / x$. Finally changing $s-1$ to $s$ we obtain $(2.5)$.

To establish (2.6) let $I$ denote the integral on the right-hand side. We shall use the following elementary (see [12, Lemma 4])

LEMMA 1. Suppose that $g(x)$ is a real-valued, integrable function on $[a, b], a$ subinterval of $[1, \infty)$, which is not necessarily finite. Then

$$
\int_{0}^{T}\left|\int_{a}^{b} g(x) x^{-s} \mathrm{~d} x\right|^{2} \mathrm{~d} t \leqslant 2 \pi \int_{a}^{b} g^{2}(x) x^{1-2 \sigma} \mathrm{d} x \quad(s=\sigma+i t, T>0, a<b) .
$$

Then by using the Cauchy-Schwarz inequality, the well-known bounds (see [10])

$$
\int_{0}^{T}\left|\zeta\left(\frac{1}{2}+i t\right)\right|^{2 k} \mathrm{~d} t \ll T(\log T)^{k^{2}} \quad(k=1,2)
$$

and (2.7) (considering $t=\Im \mathrm{m} s$ fixed and letting $T \rightarrow \infty$ ) we obtain

$$
\begin{aligned}
I^{2} & \leq \int_{-\infty}^{\infty}\left|\mathcal{M}_{1}(c+i v)\right|^{2} \mathrm{~d} v \int_{-\infty}^{\infty}\left|\mathcal{M}_{1}(1-c+\sigma+i(v+t))\right|^{2} \mathrm{~d} v \\
& \ll \int_{1}^{\infty}\left|\zeta\left(\frac{1}{2}+i x\right)\right|^{2} x^{1-2 c} \mathrm{~d} x \int_{1}^{\infty}\left|\zeta\left(\frac{1}{2}+i x\right)\right|^{4} x^{2 c-2 \sigma-1} \mathrm{~d} x \\
& \ll 1,
\end{aligned}
$$

since $1-2 c<-1,2 c-2 \sigma-1<-1$. Therefore $I$ converges absolutely and (2.6) holds, providing incidentally the analytic continuation of $\mathcal{M}_{3}(s)$ to $\sigma>1$ (this also follows directly from the defining relation (1.2)).

THEOREM 2. If $k=1,2,3,4$ and $c>1$ is fixed, then for $U \gg x$ and $\varepsilon>0$ sufficiently small we have

$$
Z^{k}(x)=\frac{1}{2 \pi i} \int_{c-i U}^{c+i U} x^{s-1} \mathcal{M}_{k}(s) \mathrm{d} s+O_{\varepsilon, k}\left(x^{c-1} U^{-\varepsilon / 2}\right) .
$$


Proof. In view of $(2.8) \mathcal{M}_{k}(s)(k \leqslant 4)$ converges absolutely for $\sigma>1$. Hence the inversion formula (1.6) and the residue theorem yield

$$
\begin{aligned}
Z^{k}(x) & =\frac{1}{2 \pi} \int_{(c)} x^{s-1} \mathcal{M}_{k}(s) \mathrm{d} s \\
& =\frac{1}{2 \pi}\left(\int_{c-i U}^{c+i U}+\int_{c-i \infty}^{c-i U}+\int_{c+i U}^{c+i \infty}\right)+O_{\varepsilon, k}\left(x^{\varepsilon}\right) \\
& =\frac{1}{2 \pi}\left(I_{1}+I_{2}+I_{3}\right)+O_{\varepsilon, k}\left(x^{\varepsilon}\right)
\end{aligned}
$$

say. Here and later $\varepsilon(>0)$ denotes constants which may be arbitrarily small, but are not necessarily the same ones at each occurrence. The $O$-term comes from the residue at $s=1$ (for $k=1$ the function $\mathcal{M}_{1}(s)$ is regular for $s=1$, while for $k=3$ very likely $\mathcal{M}_{3}(s)$ is also regular at $s=1$, but this has not been proved yet). Therefore to prove (2.9) it suffices to show that

$$
I_{3} \ll_{\varepsilon, k} x^{c-1} U^{-\varepsilon / 2},
$$

since the estimation of $I_{2}$ is analogous to the estimation of $I_{3}$. For $\sigma>1, T_{1} \leqslant$ $t \leqslant 2 T_{1}$ (with the aim of taking later $T_{1}=U, T_{1}=2 U$ etc.) we have

$$
\mathcal{M}_{k}(s)=\int_{1}^{T_{1}^{1-\varepsilon}} Z^{k}(u) \varphi(u) u^{-s} \mathrm{~d} u+\int_{\frac{1}{2} T_{1}^{1-\varepsilon}}^{\infty} Z^{k}(u)(1-\varphi(u)) u^{-s} \mathrm{~d} u=I_{4}+I_{5}
$$

say. Here $\varphi(u)(\geqslant 0)$ is a smooth function supported in $\left[1, T_{1}^{1-\varepsilon}\right]$ such that $\varphi(u)=1$ for $1 \leqslant u \leqslant \frac{1}{2} T_{1}^{1-\varepsilon}$ and

$$
\varphi^{(r)}(u) \ll_{r} T_{1}^{r(1-\varepsilon)} \quad(r=0,1,2, \ldots) .
$$

Repeated integration by parts shows that, for $N \geqslant N_{0}(\varepsilon, k)$,

$$
\begin{aligned}
I_{4} & =\frac{c_{1, k}}{s-1}+\frac{1}{s-1} \int_{1}^{T_{1}^{1-\varepsilon}} u^{1-s}\left(\varphi(u) Z^{k}(u)\right)^{\prime} \mathrm{d} u=\ldots \\
& =\frac{c_{1, k}}{s-1}+\ldots+\frac{c_{N, k}}{(s-1)^{N}}+O_{N, k}\left(T_{1}^{-\frac{1}{2} \varepsilon N}\right)
\end{aligned}
$$

since, for $\ell_{j}, m_{j} \geqslant 0$ and $\ell_{1}+\ldots+\ell_{N}=k$ (for a formula for $Z^{(m)}(x)$ see [21, p. 87]; see also [18, p. 313]),

$$
\int_{1}^{X}\left(Z^{\ell_{1}}(x)\right)^{\left(m_{1}\right)} \ldots\left(Z^{\ell_{N}}(x)\right)^{\left(m_{N}\right)} \mathrm{d} x \ll_{\varepsilon, k, N} X^{1+\varepsilon}
$$


One obtains (2.13) similarly as (2.8), using Hölder's inequality, the defining relation (1.3) and the asymptotics of the $\chi$-function. The reason that we do not have (yet) Theorem 2 for $k>4$ is essentially the fact that we do not have yet the bound

$$
\int_{0}^{T}\left|\zeta\left(\frac{1}{2}+i t\right)\right|^{m} \mathrm{~d} t \ll_{\varepsilon} T^{1+\varepsilon}
$$

for any fixed $m>4$.

Hence by the first derivative test

$$
\int_{c+i T_{1}}^{c+i 2 T_{1}} x^{s-1} I_{4} \mathrm{~d} s=\frac{c_{1, k}}{2 \pi i} \int_{c+i T_{1}}^{c+i 2 T_{1}} \frac{x^{s-1}}{s-1} \mathrm{~d} s+O\left(\frac{x^{c-1}}{T_{1}}\right)=O\left(\frac{x^{c-1}}{T_{1}}\right) .
$$

On the other hand

$$
\begin{aligned}
\int_{c+i T_{1}}^{c+i 2 T_{1}} x^{s-1} I_{5} \mathrm{~d} s & =i \int_{T_{1}}^{2 T_{1}} x^{c+i t-1}\left(\int_{\frac{1}{2} T_{1}^{1-\varepsilon}}^{\infty} Z^{k}(u)(1-\varphi(u)) u^{-c-i t} \mathrm{~d} u\right) \mathrm{d} t \\
& =i x^{c-1} \int_{\frac{1}{2} T^{1-\varepsilon}}^{\infty} Z^{k}(u)(1-\varphi(u)) u^{-c}\left(\int_{T_{1}}^{2 T_{1}} \mathrm{e}^{i t \log (x / u)} \mathrm{d} t\right) \mathrm{d} u
\end{aligned}
$$

For $T_{1} \gg x$ it follows, by direct integration, that the last integral over $t$ is bounded. Thus the last expression, for some constant $c_{k} \geqslant 0$, is

$$
\ll x^{c-1}\left(\log T_{1}\right)^{c_{k}} T_{1}^{(1-\varepsilon)(1-c)} .
$$

Therefore we have

$$
I_{3} \ll \frac{x^{c-1}}{U}+x^{c-1}(\log U)^{c_{k}} U^{(1-\varepsilon)(1-c)} \ll x^{c-1} U^{-\varepsilon / 2}
$$

if $\varepsilon>0$ is sufficiently small, and (2.9) follows. Theorem 2 is proved.

Remark 1. We can get (at least in principle) the information about the sixth moment of $\zeta\left(\frac{1}{2}+i t\right)$ from $\mathcal{M}_{3}(s)$. Namely from from (2.9) with $k=3$ or from the method of proof of Lemma 4 of [10] we get that

$$
\int_{T}^{2 T}\left|\zeta\left(\frac{1}{2}+i t\right)\right|^{6} \mathrm{~d} t \ll_{\varepsilon} T^{2 \sigma-1} \int_{1}^{T^{1+\varepsilon}}\left|\mathcal{M}_{3}(\sigma+i t)\right|^{2} \mathrm{~d} t+T^{1+\varepsilon} \quad\left(\frac{1}{2}<\sigma \leqslant 1\right)
$$

provided that $\mathcal{M}_{3}(s)$ can be continued to $\Re$ e $s \geqslant \sigma$ (and that is the catch!). Heuristically, we should be able to have $\sigma=3 / 4+\varepsilon$, and then the integral on the 
right-hand side of (2.14) should be $\ll_{\varepsilon} T^{1 / 2+\varepsilon}$, giving a weak form of the sixth moment. Note that (see [12, eq. (4.7)]) for the eighth moment we have

$$
\int_{T}^{2 T}\left|\zeta\left(\frac{1}{2}+i t\right)\right|^{8} \mathrm{~d} t \ll_{\varepsilon} T^{2 \sigma-1} \int_{1}^{T^{1+\varepsilon}}\left|\mathcal{Z}_{2}(\sigma+i t)\right|^{2} \mathrm{~d} t+T^{1+\varepsilon} \quad\left(\frac{1}{2}<\sigma \leqslant 1\right)
$$

and an analogue of (2.14) and (2.15) holds also for the mean square and fourth power of $\left|\zeta\left(\frac{1}{2}+i t\right)\right|$. In these cases, however, the results are not of particular interest, since we have precise information which has been obtained by other methods. The bounds for the sixth moment of $\left|\zeta\left(\frac{1}{2}+i t\right)\right|$ are intricately connected with the problem of the analytic continuation of $\mathcal{M}_{3}(s)$ to the region $\sigma \leqslant 1$. It should be noted that the bounds

$$
\int_{0}^{T}\left|\zeta\left(\frac{1}{2}+i t\right)\right|^{8} \mathrm{~d} t \ll_{\varepsilon} T^{1+\varepsilon}
$$

and

$$
\begin{aligned}
& \int_{T}^{2 T}\left|\mathcal{Z}_{2}(\sigma+i t)\right|^{2} \mathrm{~d} t \ll_{\varepsilon} T^{4-4 \sigma+\varepsilon} \quad\left(\frac{1}{2}<\sigma \leqslant 1\right), \\
& \int_{T}^{2 T}\left|\mathcal{Z}_{2}(\sigma+i t)\right|^{2} \mathrm{~d} t \ll_{\varepsilon} T^{2-2 \sigma+\varepsilon}+T^{-1} \quad(\sigma \geqslant 1) .
\end{aligned}
$$

are equivalent (see [12, eqs. (4.3) and (4.8)]).

The next result is a generalization of Theorem 4 of [15]. This is

THEOREM 3. In the region of absolute convergence we have

$$
\mathcal{M}_{k}^{2}(s)=2 \int_{1}^{\infty} x^{-s}\left(\int_{\sqrt{x}}^{x} Z^{k}(u) Z^{k}\left(\frac{x}{u}\right) \frac{\mathrm{d} u}{u}\right) \mathrm{d} x .
$$

Proof of Theorem 3. Set $f(x)=Z^{k}(x)$ and make the change of variables $x y=X, x / y=Y$, so that the absolute value of the Jacobian of the transformation is equal to $1 /(2 Y)$. Therefore

$$
\begin{aligned}
\mathcal{M}_{k}^{2}(s) & =\int_{1}^{\infty} \int_{1}^{\infty}(x y)^{-s} f(x) f(y) \mathrm{d} x \mathrm{~d} y \\
& =\frac{1}{2} \int_{1}^{\infty} X^{-s} \int_{1 / X}^{X} \frac{1}{Y} f(\sqrt{X Y}) f(\sqrt{X / Y}) \mathrm{d} Y \mathrm{~d} X .
\end{aligned}
$$

But as we have $(y=1 / u)$

$$
\int_{1 / x}^{x} f(\sqrt{x y}) f(\sqrt{x / y}) \frac{\mathrm{d} y}{y}=\int_{1 / x}^{1}+\int_{1}^{x}=2 \int_{1}^{x} f(\sqrt{x / u}) f(\sqrt{x u}) \frac{\mathrm{d} u}{u}
$$


we obtain that, in the region of absolute convergence, the identity

$$
\mathcal{M}_{k}^{2}(s)=\int_{1}^{\infty} x^{-s}\left(\int_{1}^{x} f(\sqrt{x y}) f(\sqrt{x / y}) \frac{\mathrm{d} y}{y}\right) \mathrm{d} x
$$

is valid. The inner integral here becomes, after the change of variable $\sqrt{x y}=u$,

$$
2 \int_{\sqrt{x}}^{x} f(u) f\left(\frac{x}{u}\right) \frac{\mathrm{d} u}{u}
$$

and (2.16) follows. The argument also shows that, for $0<a<b$ and any integrable function $f$ on $[a, b]$,

$$
\left(\int_{a}^{b} f(x) x^{-s} \mathrm{~d} x\right)^{2}=2 \int_{a^{2}}^{b^{2}} x^{-s}\left\{\int_{\sqrt{x}}^{\min (x / a, b)} f(u) f\left(\frac{x}{u}\right) \frac{\mathrm{d} u}{u}\right\} \mathrm{d} x .
$$

3. The CASES of $\mathcal{M}_{k}(s)$ When $k=1,3$

The analytic continuation of $\mathcal{M}_{k}(s)$ when $k \leqslant 4$ is interesting only when $k=$ 1,3 , since $\mathcal{M}_{2}(s) \equiv \mathcal{Z}_{1}(s), \mathcal{M}_{4}(s) \equiv \mathcal{Z}_{2}(s)$, and for $\mathcal{Z}_{1}(s), \mathcal{Z}_{2}(s)$ there is plenty of information (see Section 1). For $k>4$ there is little information available on $\mathcal{Z}_{k}(s)$. We have the following

THEOREM 4. The function $\mathcal{M}_{1}(s)$ has analytic continuation to the region $\sigma>0$, where it is regular. For fixed $\sigma$ such that $\frac{1}{4}<\sigma \leqslant \frac{5}{4}$ it satisfies

$$
\mathcal{M}_{1}(\sigma+i t) \ll_{\varepsilon} t^{\frac{3}{4}-\sigma+\varepsilon}\left(1+t^{\frac{3}{4}-\sigma}\right) \quad\left(t \geqslant t_{0}>0\right) .
$$

We also have, for fixed $\sigma$ such that $\frac{1}{2}<\sigma \leqslant 1$,

$$
\begin{aligned}
& \int_{1}^{T}\left|\mathcal{M}_{1}(\sigma+i t)\right|^{2} \mathrm{~d} t \ll_{\varepsilon} T^{2-2 \sigma+\varepsilon} \\
& \int_{1}^{T}\left|\mathcal{M}_{1}(\sigma+i t)\right|^{2} \mathrm{~d} t \gg_{\varepsilon} T^{2-2 \sigma-\varepsilon} .
\end{aligned}
$$

THEOREM 5. We have

$$
\mathcal{M}_{3}(s)=\int_{1}^{\infty} Z^{3}(x) x^{-s} \mathrm{~d} x=V_{1}(s)+V_{2}(s)
$$


say, where $V_{2}(s)$ is regular for $\sigma>3 / 4$ and for $\sigma>1$ the function

$$
V_{2}(s)=(2 \pi)^{1-s} \sqrt{\frac{2}{3}} \sum_{n=1}^{\infty} d_{3}(n) n^{-\frac{1}{6}-\frac{2 s}{3}} \cos \left(3 \pi n^{\frac{2}{3}}+\frac{1}{8} \pi\right)
$$

is regular, where $d_{3}(n)=\sum_{k \ell m=n} 1$.

Proof of Theorem 4. To prove the result on the analytic continuation of $\mathcal{M}_{1}(s)$ we use the author's method of proof [14]. By the use of Laplace transform of $\left|\zeta\left(\frac{1}{2}+i t\right)\right|^{2}$ (see e.g. [28, Theorem $\left.7.15(\mathrm{~A})\right]$ ) it was shown there that $\mathcal{Z}_{1}(s)$ has meromorphic continuation to $\mathbb{C}$. Thus let

$$
\bar{L}(s):=\int_{1}^{\infty} Z(y) \mathrm{e}^{-y s} \mathrm{~d} y, \quad L(s):=\int_{0}^{\infty} Z(y) \mathrm{e}^{-y s} d y \quad(\sigma=\Re \mathrm{e} s>0) .
$$

Then we have, by absolute convergence, taking initially $\sigma$ to be sufficiently large and making the change of variable $x y=t$,

$$
\begin{aligned}
& \int_{0}^{\infty} \bar{L}(x) x^{s-1} \mathrm{~d} x=\int_{0}^{\infty}\left(\int_{1}^{\infty} Z(y) \mathrm{e}^{-x y} \mathrm{~d} y\right) x^{s-1} \mathrm{~d} x \\
& =\int_{1}^{\infty} Z(y)\left(\int_{0}^{\infty} x^{s-1} \mathrm{e}^{-x y} \mathrm{~d} x\right) \mathrm{d} y \\
& =\int_{1}^{\infty} Z(y) y^{-s} \mathrm{~d} y \int_{0}^{\infty} \mathrm{e}^{-t} t^{s-1} \mathrm{~d} t=\mathcal{M}_{1}(s) \Gamma(s) .
\end{aligned}
$$

Since $\Gamma(s)$ has no zeros, it suffices to prove the assertion for

$$
\begin{aligned}
& \int_{0}^{\infty} \bar{L}(x) x^{s-1} \mathrm{~d} x=\int_{0}^{1} \bar{L}(x) x^{s-1} \mathrm{~d} x+\int_{1}^{\infty} \bar{L}(x) x^{s-1} \mathrm{~d} x \\
& =\int_{1}^{\infty} \bar{L}(1 / x) x^{-1-s} \mathrm{~d} x+A(s) \quad(\sigma>1),
\end{aligned}
$$

say, where

$$
A(s):=\int_{1}^{\infty} \bar{L}_{1}(x) x^{s-1} \mathrm{~d} x
$$

is an entire function. Since

$$
\bar{L}(1 / x)=L(1 / x)-\int_{0}^{1} Z(y) \mathrm{e}^{-y / x} \mathrm{~d} y \quad(x \geqslant 1),
$$

it remains to consider

$$
\begin{aligned}
\int_{1}^{\infty} \bar{L}(1 / x) x^{-s-1} \mathrm{~d} x & =\int_{1}^{\infty} L(1 / x) x^{-s-1} \mathrm{~d} x-\int_{1}^{\infty}\left(\int_{0}^{1} Z(y) \mathrm{e}^{-y / x} \mathrm{~d} y\right) x^{-s-1} \mathrm{~d} x \\
& =I_{1}(s)-I_{2}(s),
\end{aligned}
$$


say. Note that in $I_{2}(s)$ the integral over $y$ is uniformly bounded, so that $I_{2}(s)$ is regular for $\sigma>0$. To deal with $I_{1}(s)$ we shall use M. Jutila's result (see [19, Lemma 2]) that

$$
\tilde{L}(p) \ll 1, p=\frac{1}{T}+i u, T \geqslant T_{0}, 0 \leqslant u \leqslant\left(T^{1 / 2} \log T\right)^{-1}
$$

where

$$
\tilde{L}(p):=\int_{0}^{\infty} Z(t) H\left(\frac{1}{2}+i t\right) \mathrm{e}^{-p t} \mathrm{~d} t \quad(\Re \mathrm{e} p>0)
$$

with a precisely defined function $H$ which satisfies

$$
H\left(\frac{1}{2}+i t\right)=1+O\left(\frac{1}{|t|+1}\right), \quad H^{\prime}\left(\frac{1}{2}+i t\right)=O\left(\frac{1}{(|t|+1)^{2}}\right) .
$$

If we set $k(t)=1-H\left(\frac{1}{2}+i t\right)$, then

$$
I_{1}(s)=I_{3}(s)+B(s),
$$

say, where $B(s)$ is regular for $\sigma>0$ and

$$
\begin{aligned}
I_{3}(s) & :=\int_{1}^{\infty}\left(\int_{0}^{\infty} Z(t) k(t) \mathrm{e}^{-t / x} \mathrm{~d} t\right) x^{-1-s} \mathrm{~d} x \\
& =\int_{1}^{\infty} Z(t) k(t) t^{-s}\left(\int_{0}^{\infty} \mathrm{e}^{-u} u^{s-1} \mathrm{~d} u\right) \mathrm{d} t=\Gamma(s) \int_{1}^{\infty} Z(t) k(t) t^{-s} \mathrm{~d} t .
\end{aligned}
$$

Finally note that the author [13] proved that

$$
\mathcal{I}_{1}(T) \equiv F(T)=\int_{1}^{T} Z(y) \mathrm{d} y=O_{\varepsilon}\left(T^{1 / 4+\varepsilon}\right)
$$

which was improved to $F(T)=O\left(T^{1 / 4}\right)$ by M. Korolev [22], who also proved that $F(T)=\Omega_{ \pm}\left(T^{1 / 4}\right)$. M. Jutila [20] gave a different proof of the same results by establishing precise formulas for $F(T)$. Integration by parts and (3.6) show that the $\int_{1}^{\infty} Z(t) k(t) t^{-s} \mathrm{~d} t$ represents a regular function even for $\sigma>-3 / 4$, implying that $I_{3}(s)$, and consequently $\mathcal{M}_{1}(s)$, admits analytic continuation to the region $\sigma>0$, where it is regular.

To obtain the pointwise bound (3.1) we use

$$
\mathcal{M}_{1}(s)=O\left(\frac{1}{t}\right)+\int_{t^{1-\varepsilon}}^{X} Z(x) x^{-s} \mathrm{~d} x+\int_{X}^{\infty} Z(x) x^{-s} \mathrm{~d} x
$$


which is valid initially for $\sigma>1$ and where $X(\gg t)$ is a parameter to be chosen a little later. One obtains (3.7) by using the argument in (2.12). Integration by parts and (3.6) show that

$$
\int_{X}^{\infty} Z(x) x^{-s} \mathrm{~d} x \ll \varepsilon t^{1+\varepsilon} X^{1 / 4-\sigma} \quad\left(\sigma>1 / 4, X \ll t^{C}\right) .
$$

The remaining integral in (3.7) is split into $O(\log t)$ integrals of the form

$$
\begin{aligned}
& \int_{Y}^{Y^{\prime}} Z(x) x^{-s} \mathrm{~d} x \\
& =2 \int_{Y}^{Y^{\prime}} \sum_{n \leqslant \sqrt{\frac{x}{2 \pi}}} n^{-1 / 2} \cos \left(x \log \frac{\sqrt{x /(2 \pi)}}{n}-\frac{1}{2} x-\frac{\pi}{8}\right) x^{-s} \mathrm{~d} x+O\left(\int_{Y}^{Y^{\prime}} x^{-1 / 4-\sigma} \mathrm{d} x\right),
\end{aligned}
$$

where $Y<Y^{\prime} \leqslant 2 Y$, and we used a version of the classical Riemann-Siegel formula (see e.g., [10, eq. (4.5)]) for $Z(t)$. Interchanging summation and integration it is seen that the expression on the right-hand side above is

$$
2 \sum_{n \leqslant \sqrt{\frac{Y^{\prime}}{2 \pi}}} n^{-1 / 2} \int_{\max \left(Y, 2 \pi n^{2}\right)}^{Y^{\prime}} x^{-\sigma} \mathrm{e}^{i F_{ \pm}(x)} \mathrm{d} x+O\left(Y^{3 / 4-\sigma}\right),
$$

with

$$
\begin{aligned}
& F_{ \pm}(x):=x \log \frac{\sqrt{x /(2 \pi)}}{n}-\frac{1}{2} x-\frac{\pi}{8} \pm t \log x, \\
& F_{ \pm}^{\prime}(x)=\log \frac{\sqrt{x /(2 \pi)}}{n} \pm \frac{t}{x}, \quad F_{ \pm}^{\prime \prime}(x)=\frac{1}{2 x} \mp \frac{t}{x^{2}} .
\end{aligned}
$$

Consider the contribution of $F_{+}(x)$, when $F_{+}^{\prime}(x)>0$. If $Y>4 t$ then $1 /(2 x)>$ $2 t /\left(x^{2}\right)$, hence by the second derivative test (Lemma 2.1 of [10]) the sum in (3.9) is $\ll Y^{3 / 4-\sigma}$. If $Y<t / 2$ then $t /\left(x^{2}\right)>1 / x$, hence again by the second derivative test we obtain a contribution which is

$$
\ll Y t^{-1 / 2} \cdot Y^{1 / 4-\sigma} \ll Y^{3 / 4-\sigma} .
$$

If $t / 2 \leqslant Y \leqslant 4 t$, then $F_{+}^{\prime}(x) \gg 1$, hence by the first derivative test we obtain again a contribution which is $\ll Y^{3 / 4-\sigma}$. A similar analysis holds for the contribution of $F_{-}(x)$, when $F_{-}^{\prime \prime}(x) \gg 1 / x$. Therefore we have

$$
\int_{Y}^{Y^{\prime}} Z(x) x^{-s} \mathrm{~d} x \ll_{\varepsilon} t^{(3 / 4-\sigma)(1-\varepsilon)}+X^{3 / 4-\sigma} .
$$


Choosing $X=t^{2}$ and noting that $t^{3 / 2-2 \sigma} \gg t^{-1}$ for $\sigma \leqslant 5 / 4$ we obtain (3.1) from (3.8) and (3.10).

Remark 2. For $\sigma>1 / 2$ note that the bound in (3.1) is better than the bound

$$
\mathcal{Z}_{1}(\sigma+i t) \ll_{\varepsilon} t^{1-\sigma+\varepsilon} \quad\left(0 \leqslant \sigma \leqslant 1, t \geqslant t_{0}>0\right),
$$

proved in [18], and for $\sigma>2 / 3$ the bound with the exponent $5 / 6-\sigma+\varepsilon$ proved by M. Jutila [19].

The mean square bound (3.2) for $\mathcal{M}_{1}$ follows by the method of proof of (see $[18$, eq. (3.7)])

$$
\int_{1}^{T}\left|\mathcal{Z}_{1}(\sigma+i t)\right|^{2} \mathrm{~d} t \ll_{\varepsilon} T^{2-2 \sigma+\varepsilon} \quad(1 / 2 \leqslant \sigma \leqslant 1),
$$

where instead of Atkinson's formula [1] for the error term in the mean square formula for $\left|\zeta\left(\frac{1}{2}+i t\right)\right|$ we use Theorem 1 of M. Jutila [20], which is the analogue of Atkinson's formula for $Z(t)$, so that there is no need to repeat the details. In this way it is seen that for the mean square we do not obtain a better estimate for $\mathcal{M}_{1}$ than the one derived for $\mathcal{Z}_{1}$. In fact it was proved (see [12] and [14]) that

$$
\int_{1}^{T}\left|\mathcal{Z}_{k}(\sigma+i t)\right|^{2} \mathrm{~d} t \gg_{\varepsilon} T^{2-2 \sigma-\varepsilon} \quad\left(k=1,2 ; \frac{1}{2}<\sigma \leqslant 1\right),
$$

and the lower bound in (3.3) is the analogue of (3.12) for $\mathcal{M}_{1}$. The proof also bears similarities to the proofs of (3.12), but we shall give here a sketch of the proof. From Theorem 2 (with $c=\frac{5}{4}, U=X, x \asymp X$ ) we have

$$
\begin{aligned}
Z(x) & =\frac{1}{2 \pi i} \int_{\frac{5}{4}-i X}^{\frac{5}{4}+i X} x^{s-1} \mathcal{M}_{1}(s) \mathrm{d} s+O\left(X^{-1 / 4}\right) \\
& =\frac{1}{2 \pi i} \int_{c-i X}^{c+i X} x^{s-1} \mathcal{M}_{1}(s) \mathrm{d} s+O\left(\int_{c}^{\frac{5}{4}} x^{\sigma-1}\left|\mathcal{M}_{1}(c+i X)\right| \mathrm{d} \sigma\right)+O\left(X^{-1 / 4}\right) .
\end{aligned}
$$

Now we use the bound (3.1) to obtain that the error terms above are

$$
\ll_{\varepsilon} X^{\frac{1}{2}-c+\varepsilon}+X^{\varepsilon-\frac{1}{4}} \ll_{\varepsilon} X^{-\varepsilon} \quad\left(c \geqslant \frac{1}{2}+2 \varepsilon\right) .
$$

Therefore

$$
\int_{X}^{2 X} Z^{2}(x) \mathrm{d} x \ll \int_{X}^{2 X}\left|\int_{c-i X}^{c+i X} x^{s-1} \mathcal{M}_{1}(s) \mathrm{d} s\right|^{2} \mathrm{~d} x+X^{1-2 \varepsilon}
$$


Since $Z^{2}(x)=\left|\zeta\left(\frac{1}{2}+i x\right)\right|^{2}$ and $\int_{X}^{2 X}\left|\zeta\left(\frac{1}{2}+i x\right)\right|^{2} \mathrm{~d} x \gg X \log X$, it follows that

$$
X \log X \ll \int_{X / 2}^{5 X / 2} \varphi(x)\left|\int_{1}^{X} x^{c+i t-1} \mathcal{M}_{1}(c+i t) \mathrm{d} t\right|^{2} \mathrm{~d} x
$$

as

$$
\int_{0}^{1} x^{s-1} \mathcal{M}_{1}(s) \mathrm{d} s \ll 1 \quad\left(x \asymp X, \frac{1}{2}<c \leqslant 1\right) .
$$

Here $\varphi(x)(\geqslant 0)$ is a smooth function supported in $[X / 2,5 X / 2]$ and equal to unity in $[X, 2 X]$. When we develop the square on right-hand side of (3.13) and integrate sufficiently many times by parts we obtain that

$$
\begin{aligned}
X \log X & \ll \int_{X / 2}^{5 X / 2} x^{2 c-2} \int_{1}^{X} \int_{1,|u-t| \leqslant X^{\varepsilon}}^{X}\left|\mathcal{M}_{1}(c+i t) \mathcal{M}_{1}(c+i u)\right| \mathrm{d} u \mathrm{~d} t \mathrm{~d} x \\
& \ll X^{2 c-1} \int_{1}^{X} \int_{1,|u-t| \leqslant X^{\varepsilon}}^{X}\left(\left|\mathcal{M}_{1}(c+i t)\right|^{2}+\left|\mathcal{M}_{1}(c+i u)\right|^{2}\right) \mathrm{d} u \mathrm{~d} t \\
& \ll \varepsilon X^{2 c-1+\varepsilon} \int_{1}^{X}\left|\mathcal{M}_{1}(c+i t)\right|^{2} \mathrm{~d} t,
\end{aligned}
$$

since the contribution of $|u-t| \geqslant X^{\varepsilon}$ will be negligibly small. This implies the assertion (3.3) with $\sigma=c \geqslant \frac{1}{2}+2 \varepsilon$.

Proof of Theorem 5. Note that from Theorem 5 of Section 4 (with $k=3$ ) we obtain (cf. (1.4))

$$
\mathcal{I}_{3}(x)=2 \pi \sqrt{\frac{2}{3}} \sum_{n \leqslant\left(\frac{x}{2 \pi}\right)^{3 / 2}} d_{3}(n) n^{-\frac{1}{6}} \cos \left(3 \pi n^{\frac{2}{3}}+\frac{1}{8} \pi\right)+O_{\varepsilon}\left(x^{3 / 4+\varepsilon}\right) .
$$

Inserting (3.14) in (1.5) we see that

$$
\mathcal{Z}_{3}(s)=V_{1}(s)+V_{2}(s)
$$

where $V_{2}(s)$ (coming from the error term) is obviously regular for $\sigma>3 / 4$ and satisfies $V_{2}(s)=O(|s|+1)$. Therefore the main problem is the analytic continuation of

$$
V_{1}(s):=2 \pi \sqrt{\frac{2}{3}} s \int_{1}^{\infty} x^{-s-1} \sum_{n \leqslant\left(\frac{x}{2 \pi}\right)^{3 / 2}} d_{3}(n) n^{-\frac{1}{6}} \cos \left(3 \pi n^{\frac{2}{3}}+\frac{1}{8} \pi\right) \mathrm{d} x .
$$


If in (3.15) we invert the order of summation and integration we get

$$
\begin{aligned}
V_{1}(s) & =-2 \pi \sqrt{\frac{2}{3}} \sum_{n=1}^{\infty} d_{3}(n) n^{-\frac{1}{6}} \cos \left(3 \pi n^{\frac{2}{3}}+\frac{1}{8} \pi\right) \int_{2 \pi n^{2 / 3}}^{\infty} \mathrm{d}\left(x^{-s}\right) \\
& =(2 \pi)^{1-s} \sqrt{\frac{2}{3}} \sum_{n=1}^{\infty} d_{3}(n) n^{-\frac{1}{6}-\frac{2 s}{3}} \cos \left(3 \pi n^{\frac{2}{3}}+\frac{1}{8} \pi\right) .
\end{aligned}
$$

The series in (3.16) converges absolutely for $\sigma>5 / 4$. This is trivial, and we seek a better result. By considering the portion of the series in (3.16) over $[X, 2 X]$ (for large $X$ and $s=\sigma+i t$ fixed) we want to show that it is $\ll_{\varepsilon} X^{-\varepsilon}$, which provides then the desired analytic continuation to the right of the $\sigma$-line. By using the Stieltjes integral representation and then integration by parts, we are led to two integrals, of which the relevant one is

$$
J(s ; X):=\int_{X}^{2 X} \Delta_{3}(x) x^{-\frac{1}{2}-\frac{2 s}{3}} \cos \left(3 \pi x^{\frac{2}{3}}+\frac{1}{8} \pi\right) \mathrm{d} x .
$$

On applying the truncated Perron inversion formula (see e.g., [10, Appendix]) we have

$$
\Delta_{3}(x)=\frac{1}{2 \pi i} \int_{\frac{1}{2}-i X}^{\frac{1}{2}+i X} \frac{\zeta^{3}(w) x^{w}}{w} \mathrm{~d} w+O_{\varepsilon}\left(X^{\varepsilon}\right) \quad(X \leqslant x \leqslant 2 X),
$$

where as usual $\Delta_{3}(x)$ is the error term in the asymptotic formula for the summatory function of $d_{3}(n)$. The error term in (3.18) contributes to the integral in (3.17) $\ll_{\varepsilon} X^{\frac{1}{2}-\frac{2 \sigma}{3}+\varepsilon} \ll_{\varepsilon} X^{-\varepsilon}$ for $\sigma>3 / 4$. The main term in (3.18) produces

$$
\frac{1}{2 \pi i} \int_{\frac{1}{2}-i X}^{\frac{1}{2}+i X} \frac{\zeta^{3}(w)}{w}\left(\int_{X}^{2 X} x^{-2 \sigma / 3} \exp \left(i F_{ \pm}(x)\right) \mathrm{d} x\right) \mathrm{d} w
$$

where

$$
w=\frac{1}{2}+i v, s=\sigma+i t, F_{ \pm}(x):=(v-(2 t) / 3) \log x \pm 3 \pi x^{2 / 3} .
$$

Note that the saddle point

$$
x_{0}=\left(\frac{|v-(2 t) / 3|}{2 \pi}\right)^{3 / 2} \in[X, 2 X] \quad\left(\text { for } v \asymp X^{2 / 3}\right),
$$

in which case $\left|F_{ \pm}\left(x_{0}\right)\right|^{-1 / 2} \asymp X^{2 / 3}$. Hence by the saddle-point method the total contribution to $(3.18)$ is $\ll_{\varepsilon} X^{(2 / 3)(1-\sigma)+\varepsilon}$, and this provides the desired analytic continuation of $\mathcal{Z}_{3}(s)$ only to $\sigma>1$ as before. One can make the calculation of 
(3.17) simpler by making the change of variable $x^{2 / 3}=y$, after $\Delta_{3}(x)$ is replaced by (3.18). However at present I do not see any better way to tackle the problem of the analytic continuation of $\mathcal{Z}_{3}(s)$, although I feel that it can be done.

Remark 3. It is curious that obviously the shapes of $\mathcal{M}_{k}(s)$ for $k=1,2,3,4$ (the cases when we know something relevant) are totally different! The fact that $Z(x)$ is an oscillating function, while $\left|\zeta\left(\frac{1}{2}+i x\right)\right|$ is non-negative is reflected in what we expect: $\mathcal{M}_{2 \ell}(s)=\mathcal{Z}_{\ell}(s)$ should have a pole of order $\ell^{2}+1$ at $s=1$, while $\mathcal{M}_{2 \ell-1}(s)$ should be regular at $s=1$, at least for $1 \leqslant \ell \leqslant 4$.

\section{The CUBic Moment of $Z(t)$}

Let, as usual, $d_{k}(n)$ denote the number of ways $n$ can be written as a product of $k$ factors, so that $d_{k}(n)$ is the multiplicative function generated by $\zeta^{k}(s)$. In particular, $d_{1}(n) \equiv 1$ and $d_{2}(n) \equiv d(n)$, the number of divisors of $n$. To prove the second part of Theorem 4 we need the case $k=3$ of

THEOREM 6. For fixed $k=1,2,3,4$ we have

$$
\begin{aligned}
\int_{T}^{2 T} Z^{k}(t) \mathrm{d} t & =2 \pi \sqrt{\frac{2}{k}} \sum_{\left(\frac{T}{2 \pi}\right)^{k / 2} \leqslant n \leqslant\left(\frac{T}{\pi}\right)^{k / 2}} d_{k}(n) n^{-\frac{1}{2}+\frac{1}{k}} \cos \left(k \pi n^{\frac{2}{k}}+\frac{1}{8}(k-2) \pi\right) \\
& +\ldots+O_{\varepsilon}\left(T^{k / 4+\varepsilon}\right),
\end{aligned}
$$

where $+\ldots$ denotes terms similar to the one on the right-hand side of (4.1), with the similar cosine term, but of a lower order of magnitude.

Proof of Theorem 6. For $Z^{k}(t)$ we shall use a finite, smoothed sum, which is a form of the so-called approximate functional equation. One could also use a form of the approximate functional equation which comes from the so-called "reflection principle" (see e.g., Chapter 4 of [10]). However, to have a symmetric expression we shall use essentially a variant of the approximate functional equation for $\zeta^{k}(s)$ which is to be found in Chapter 4 of [11]. To this end let $\rho(x)$ be a non-negative, smooth function supported in $[0,2]$, such that $\rho(x)=1$ for $0 \leqslant x \leqslant 1 / b$ for a fixed constant $b>1$, and $\rho(x)+\rho(1 / x)=1$ for all $x$ (an explicit construction of $\rho(x)$ was given in [11]). Let $\tau=\tau(k, t)$ be defined as

$$
\log \tau=-k \frac{\chi^{\prime}\left(\frac{1}{2}+i t\right)}{\chi\left(\frac{1}{2}+i t\right)}
$$

We write

$$
\chi(s)=\pi^{s-1 / 2} \frac{\Gamma\left(\frac{1}{2}-\frac{1}{2} s\right)}{\Gamma\left(\frac{1}{2} s\right)}=\left(\frac{2 \pi}{t}\right)^{\sigma+i t-1 / 2} \mathrm{e}^{i(t+\pi / 4)}\left(1+O\left(\frac{1}{t}\right)\right)
$$


by using Stirling's formula for the gamma-function. Here $s=\sigma+i t, 0 \leqslant \sigma \leqslant$ $1, t \geqslant t_{0}>0$, and note that the $O$-term in (4.3) admits an asymptotic expansion in terms of negative powers of $t$. Therefore

$$
\frac{\chi^{\prime}\left(\frac{1}{2}+i t\right)}{\chi\left(\frac{1}{2}+i t\right)}=\log \left(\frac{2 \pi}{t}\right)+O\left(\frac{1}{t^{2}}\right)
$$

and we obtain

$$
\tau=\left(\frac{t}{2 \pi}\right)^{k}\left(1+O\left(\frac{1}{t^{2}}\right)\right)
$$

and again the $O$-term in (4.4) admits an asymptotic expansion in terms of negative powers of $t$. In the course of the proof of Theorem 5.2 of [11] it was shown that $\left(1 \ll x, y \ll t^{k}, x y=\tau, s=\sigma+i t, t \geqslant t_{0}>0,0 \leqslant \sigma \leqslant 1\right)$

$$
\zeta^{k}(s)=\sum_{n=1}^{\infty} d_{k}(n) \rho\left(\frac{n}{x}\right) n^{-s}+\chi^{k}(s) \sum_{n=1}^{\infty} d_{k}(n) \rho\left(\frac{n}{y}\right) n^{s-1}+R_{k}(t)
$$

say, where for any fixed $A>0$

$$
R_{k}(t) \ll_{\varepsilon} t^{-A}+t^{\varepsilon-1} \int_{-t^{\varepsilon}}^{t^{\varepsilon}}|\zeta(\sigma+i t-\varepsilon+i v)|^{k} \mathrm{~d} v .
$$

Thus from (4.3)-(4.6) we obtain, with $b=2, \sigma=\frac{1}{2}, x=y=\sqrt{\tau}, t \geqslant t_{0}>0$ the following

LEMMA 2. We have

$$
\begin{aligned}
\int_{T}^{2 T} Z^{k}(t) \mathrm{d} t & =2 \int_{T}^{2 T} \sum_{n \leqslant 2 \sqrt{\tau}} \rho\left(\frac{n}{\sqrt{\tau}}\right) d_{k}(n) n^{-1 / 2} \cos F_{k}(t) \mathrm{d} t+\ldots \\
& +O\left(T^{\varepsilon-1} \int_{T / 2}^{5 T / 2}\left|\zeta\left(\frac{1}{2}+i t\right)\right|^{k} \mathrm{~d} t\right)
\end{aligned}
$$

where $\tau$ is given by (4.2) and (4.4), +.. denotes terms similar to the one on the right-hand side of (4.7), but of a lower order of magnitude, and where

$$
F_{k}(t):=t \log \left\{\frac{\left(\frac{t}{2 \pi}\right)^{k / 2}}{n}\right\}-\frac{k t}{2}-\frac{k \pi}{8}
$$


To evaluate the left-hand side of (4.7) we write first

$$
\begin{aligned}
& 2 \int_{T}^{2 T} \sum_{n \leqslant 2 \sqrt{\tau}} \rho\left(\frac{n}{\sqrt{\tau}}\right) d_{k}(n) n^{-1 / 2} \cos F_{k}(t) \mathrm{d} t \\
& =2 \sum_{n \leqslant T_{0}} d_{k}(n) n^{-1 / 2} \Re \mathrm{e}\left\{\int_{T_{1}}^{2 T} \rho\left(\frac{n}{\sqrt{\tau}}\right) \mathrm{e}^{i F_{k}(t)} \mathrm{d} t\right\} .
\end{aligned}
$$

Here

$$
T_{0}=2 \sqrt{\tau(k, 2 T)}=2\left(\frac{T}{\pi}\right)^{k / 2}\left(1+O\left(\frac{1}{T^{2}}\right)\right), \quad T_{1}=\max \left(T, \tau^{-1}\left(k,(n / 2)^{2}\right)\right.
$$

where $\tau^{-1}$ is the inverse function of $\tau$, so that

$$
\tau^{-1}\left(k,(n / 2)^{2}\right)=2 \pi\left(\frac{n}{2}\right)^{2 / k}\left(1+O\left(\frac{1}{T^{2}}\right)\right) .
$$

Now we split the range of summation over $n$ on the right hand side of (4.9) as follows. Let

$$
\begin{aligned}
& I_{1}:=\left[1,\left(\frac{T}{2 \pi}\right)^{k / 2}-T^{k / 2-1 / 2+\varepsilon}\right) \\
& I_{2}:=\left[\left(\frac{T}{2 \pi}\right)^{k / 2}-T^{k / 2-1 / 2+\varepsilon},\left(\frac{T}{2 \pi}\right)^{k / 2}+T^{k / 2-1 / 2+\varepsilon}\right), \\
& I_{3}:=\left[\left(\frac{T}{2 \pi}\right)^{k / 2}+T^{k / 2-1 / 2+\varepsilon},\left(\frac{T}{\pi}\right)^{k / 2}-T^{k / 2-1 / 2+\varepsilon]},\right. \\
& I_{4}:=\left(\left(\frac{T}{\pi}\right)^{k / 2}-T^{k / 2-1 / 2+\varepsilon},\left(\frac{T}{\pi}\right)^{k / 2}+T^{k / 2-1 / 2+\varepsilon}\right] \\
& I_{5}:=\left(\left(\frac{T}{\pi}\right)^{k / 2}+T^{k / 2-1 / 2+\varepsilon}, T_{0}\right]
\end{aligned}
$$

In the integrals over where $n \in I_{1}$ and $n \in I_{5}$ we integrate by parts, writing

$$
\int \rho\left(\frac{n}{\sqrt{\tau}}\right) \mathrm{e}^{i F_{k}(t)} \mathrm{d} t=\int \frac{\rho\left(\frac{n}{\sqrt{\tau}}\right)}{i \log \left\{(t / 2 \pi)^{k / 2} / n\right\}} \mathrm{de}^{i F_{k}(t)} .
$$


Note that the derivatives of $\rho(n / \sqrt{\tau})$, considered as a function of $t$, decrease after each integration by parts by a factor of $t$, while in $\sum_{n \in I_{1}} \int$ we have

$$
\begin{aligned}
\left(\frac{1}{\log \left\{(t / 2 \pi)^{k / 2} / n\right\}}\right)^{\prime} & =-\frac{2}{k t \log ^{2}\left\{(t / 2 \pi)^{k / 2} / n\right\}} \\
& \ll_{\varepsilon} \frac{1}{T \log ^{2}\left\{\frac{C T^{k / 2}}{T^{k / 2}+O\left(T^{k / 2-1 / 2+\varepsilon}\right)}\right\}} \ll_{\varepsilon} T^{-2 \varepsilon} .
\end{aligned}
$$

Therefore if we integrate by parts sufficiently many times, the contribution will be negligible. The sums over the integrated terms are essentially partial sums of $\zeta^{k}\left(\frac{1}{2}+i u\right), u \asymp T$, when we remove the monotonic coefficients $\rho$ from the sums over $n$ by partial summation. The resulting sums are bounded by Perron's inversion formula (see e.g., the Appendix of [10]). Since $\zeta\left(\frac{1}{2}+i t\right) \ll t^{c}$ for some $c<1 / 6$ (ibid., Chapter 7), we see that

$$
\sum_{n \in I_{1}}+\sum_{n \in I_{5}} \ll T^{k / 6}
$$

Note that (cf. (4.8))

$$
F_{k}^{\prime}(t)=\log \left\{(t / 2 \pi)^{k / 2} / n\right\}, \quad F_{k}^{\prime \prime}(t)=k /(2 t) .
$$

The integrals when $n \in I_{2} \cup I_{4}$ are estimated as $\ll T^{1 / 2}$ by the second derivative test (see Chapter 2 of [10]), and then trivial estimation gives

$$
\sum_{n \in I_{2}}+\sum_{n \in I_{4}} \ll_{\varepsilon} T^{1 / 2} T^{k / 2-1 / 2+\varepsilon} T^{-k / 4}=T^{k / 4+\varepsilon}
$$

Finally when, in (4.9), we have $n \in I_{3}$, then the saddle point (root of $F_{k}^{\prime}(t)=0$ ), namely

$$
t_{0} \equiv c_{n}:=2 \pi n^{2 / k}
$$

lies in $\left[T_{1}, 2 T\right]$. For $\int_{T_{1}}^{2 T}$ we could use a general result on exponential integrals, such as the following [21, Lemma III.2], which says that

$$
\begin{aligned}
& \int_{a}^{b} \varphi(x) \exp (2 \pi i f(x)) \mathrm{d} x=\frac{\varphi(c)}{\sqrt{f^{\prime \prime}(c)}} \mathrm{e}^{2 \pi i f(c)+\pi i / 4}+O\left(H A U^{-1}\right) \\
& +O\left(H \min \left(\left|f^{\prime}(a)\right|^{-1}, \sqrt{A}\right)+O\left(H \min \left(\left|f^{\prime}(b)\right|^{-1}, \sqrt{A}\right)\right.\right.
\end{aligned}
$$


if $f^{\prime}(c)=0, a \leqslant c \leqslant b$, and the following conditions hold: $f(x) \in C^{4}[a, b], \varphi(x) \in$ $C^{2}[a, b], f^{\prime \prime}(x)>0$ in $[a, b], f^{\prime \prime}(x) \asymp A^{-1}, f^{(3)}(x) \ll A^{-1} U^{-1}, f^{(4)}(x) \ll A^{-1} U^{-2}$, $\varphi^{(r)}(x) \ll H U^{-r}(r=0,1,2)$ in $[a, b], 0<H, A<U, 0<b-a \leqslant U$. In our case $f(x)=\frac{1}{2 \pi} F_{k}(x)$, so that $f^{\prime \prime}(c)=k /(4 \pi c)$, and

$$
\frac{\varphi\left(c_{n}\right)}{\sqrt{f^{\prime \prime}\left(c_{n}\right)}} \mathrm{e}^{2 \pi i f\left(c_{n}\right)+\pi i / 4}=\pi \sqrt{\frac{2}{k}} n^{\frac{1}{k}} \exp \left(-k \pi i n^{\frac{2}{k}}+\frac{(2-k) \pi i}{8}\right)\left\{1+O\left(\frac{1}{T^{2}}\right)\right\} .
$$

But, as remarked in [13], in our case the last two error terms in (4.17) are large, and thus it is more expedient to carry out the evaluation by the saddle point technique directly, that is, by using a suitable contour in the complex plane.

To this end, if $T_{1}=T$ (the other case is similar) let $\mathcal{L}_{1}$ be the segment $T-i v(0 \leqslant$ $\left.v \leqslant \frac{1}{\sqrt{2}} T^{1-\varepsilon}\right), \mathcal{L}_{2}$ is the segment $x-i \frac{1}{\sqrt{2}} T^{1-\varepsilon}\left(0 \leqslant x \leqslant c_{n}-\frac{1}{\sqrt{2}} T^{1-\varepsilon}\right), \mathcal{L}_{3}$ is the segment $c_{n}+v \mathrm{e}^{\frac{1}{4} \pi i},-\frac{1}{\sqrt{2}} T^{1-\varepsilon} \leqslant v \leqslant \frac{1}{\sqrt{2}} T^{1-\varepsilon}, \mathcal{L}_{4}$ is the segment $x+i \frac{1}{\sqrt{2}} T^{1-\varepsilon}\left(c_{n}+\right.$ $\left.\frac{1}{\sqrt{2}} T^{1-\varepsilon} \leqslant x \leqslant 2 T\right)$, and finally $\mathcal{L}_{4}$ is the segment joining the points $2 T+i \frac{1}{\sqrt{2}} T^{1-\varepsilon}$ and $2 T$.

As a simplification we develop $\rho(n / \sqrt{\tau})$ by Taylor's formula at the point $t_{0}=c_{n}$ when $t \in\left[c_{n}-T^{1-\varepsilon}, c_{n}+T^{1-\varepsilon}\right]$, and at other appropriate points for other values of $t$. An alternative approach is to use the Mellin inversion formula:

$$
\rho(x)=\frac{1}{2 \pi} \int_{d-i \infty}^{d+i \infty} R(s) x^{-s} \mathrm{~d} s(d>0), \quad R(s)=\int_{0}^{\infty} \rho(x) x^{s-1} \mathrm{~d} x .
$$

The function $R(s)$ is odd, and of fast decay.

As already noted the derivatives of $\rho(n / \sqrt{\tau})$, considered as a function of $t$, decrease each time by a factor of $t$. Since the length of the interval is $2 T^{1-\varepsilon}$, it is possible to take finitely many terms in Taylor's formula so that the total contribution of the error term is negligible, namely $\ll 1$. The remaining integrals will be all of the same type, with the same exponential factor, and the largest one will be the first one, namely the one with $\left(c_{n}=2 \pi n^{2 / k}\right)$

$$
\rho\left(\frac{n}{\sqrt{\tau\left(k, c_{n}\right)}}\right)=\rho\left(\frac{n}{n\left(1+O\left(T^{-2}\right)\right.}\right)=\rho(1)+O\left(T^{-2}\right)=\frac{1}{2}+O\left(T^{-2}\right),
$$

since $\rho(x)+\rho(1 / x)=1$. Here actually the $O$-term above has an asymptotic expansion. After that we replace the subinterval integral over $\left[T_{1}, 2 T\right]$, by Cauchy's theorem, by $\cup_{j=1}^{5} \int_{\mathcal{L}_{j}}$. Therefore

$$
\begin{aligned}
& 2 \sum_{n \in I_{3}} d_{k}(n) n^{-1 / 2} \Re \mathrm{e}\left\{\int_{T_{1}}^{2 T} \rho\left(\frac{n}{\sqrt{\tau}}\right) \mathrm{e}^{i F_{k}(t)} \mathrm{d} t\right\} \\
& =2 \sum_{n \in I_{3}} d_{k}(n) n^{-1 / 2} \Re \mathrm{e}\left\{\bigcup_{j=1}^{5} \int_{\mathcal{L}_{j}} \mathrm{e}^{i F_{k}(z)} \mathrm{d} z\right\}+\ldots,
\end{aligned}
$$


where $+\ldots$ has the same meaning as before. On $\mathcal{L}_{3}$ we have $\left(\right.$ since $\left.F_{k}^{\prime}\left(c_{n}\right)=0\right)$

$$
i F_{k}(z)=i F_{k}\left(c_{n}\right)+i \frac{v^{2}}{2 !} \mathrm{e}^{\frac{1}{2} \pi i} F_{k}^{\prime \prime}\left(c_{n}\right)+i \frac{v^{3}}{3 !} \mathrm{e}^{\frac{3}{4} \pi i} F_{k}^{\prime \prime \prime}\left(c_{n}\right)+i \frac{v^{4}}{4 !} F_{k}^{(4)}\left(c_{n}\right)+\cdots
$$

Note that, since $v \ll T^{1-\varepsilon}$,

$$
v^{m} F_{k}^{(m)}\left(c_{n}\right) \ll_{m, \varepsilon} T^{m(1-\varepsilon)} T^{1-m}=T^{1-m \varepsilon} \quad(m>1) .
$$

Hence if we choose $M=M(k, \varepsilon)$ sufficiently large, then (4.7) shows that the terms of the series in (4.20) for $m>M$, on using $\exp z=1+O(|z|)$ for $|z| \leqslant 1$, will make a negligible contribution. We have

$$
\exp \left(i F_{k}(z)\right)=\exp \left(i F_{k}\left(c_{n}\right)\right) \exp \left(-\frac{1}{2} v^{2} F^{\prime \prime}\left(c_{n}\right)\right) \exp \left(\sum_{m=3}^{M} d_{m} v^{m} F^{(m)}\left(c_{n}\right)\right)
$$

with $d_{m}=\exp \left((m+2) \frac{\pi i}{4}\right) / m$ !. The last exponential factor is expanded by Taylor's series, and again the terms of the series (with $v^{m}$ ) for large $m$ will make a negligible contribution. In the remaining terms we restore integration over $v$ to the whole real line, making a very small error. Then we use the classical integral (see e.g., the Appendix of [10])

$$
\int_{-\infty}^{\infty} \exp \left(A x-B x^{2}\right) \mathrm{d} x=\sqrt{\frac{\pi}{B}} \exp \left(\frac{A^{2}}{4 B}\right) \quad(\Re \mathrm{e} B>0) .
$$

By differentiating (4.22) as a function of $A$ we may explicitly evaluate integrals of the type

$$
\int_{-\infty}^{\infty} x^{2 m} \exp \left(-B x^{2}\right) \mathrm{d} x \quad(\Re \mathrm{e} B>0, m=0,1,2, \ldots) .
$$

It transpires that the largest contribution $(=\sqrt{\pi})$ will come from the integral with $m=0$, which will coincide with the contribution of the main term in (4.17).

It remains to deal with the remaining integrals over $\mathcal{L}_{j}$. The integrals over $\mathcal{L}_{1}$ and $\mathcal{L}_{5}$, and likewise the integrals over $\mathcal{L}_{2}$ and $\mathcal{L}_{4}$ are estimated analogously. On $\mathcal{L}_{4}$ we have

$$
z=x+i H, c_{n}+\frac{H}{\sqrt{2}} \leqslant x \leqslant 2 T, H=T^{1-\varepsilon} .
$$

On using Taylor's formula we obtain

$$
\exp \left(i F_{k}(z)\right)=\exp \left(i F_{k}(x)-i \frac{H^{2}}{2 !} F_{k}^{\prime \prime}(x)+\ldots\right) \exp \left(-H F_{k}^{\prime}(x)+\frac{H^{3}}{3 !} F_{k}^{\prime \prime \prime}(x)-\ldots\right)
$$


Similarly as in (4.21) it follows that we may truncate the series after a finite number of terms with a negligible error. Observe that the real-valued term in the exponential is negative, and that the derivative of the imaginary part is dominated by

$$
\begin{aligned}
F_{k}^{\prime}(x) & =\log \frac{\left(\frac{x}{2 \pi}\right)^{k / 2}}{n} \geqslant \log \frac{\left(n^{2 / k}+H / \sqrt{2}\right)^{k / 2}}{n} \\
& =\log \left(1+\frac{H}{\sqrt{8} \pi n^{2 / k}}\right)^{k / 2} \geqslant A_{k} H T^{-1}=A_{k} T^{-\varepsilon}
\end{aligned}
$$

for some constant $A_{k}>0$. Hence by the first derivative test the total contribution of such terms is

$$
\ll_{\varepsilon} T^{k / 4+\varepsilon} \text {. }
$$

On $\mathcal{L}_{5}$ we have $z=2 T+i y, 0 \leqslant y \leqslant H, H=T^{1-\varepsilon}$. This gives

$$
i F_{k}(z)=i F_{k}(2 T)-y F_{k}^{\prime}(2 T)-i \frac{y^{2}}{2 !} F_{k}^{\prime \prime}(2 T)+\frac{y^{3}}{3 !} F_{k}^{\prime \prime \prime}(T)-\ldots,
$$

where, as before, we may truncate the series after a finite number of terms with a negligible error. Therefore the integral over $\mathcal{L}_{5}$ becomes

$$
i \mathrm{e}^{i F_{k}(2 T)} \int_{0}^{H} \mathrm{e}^{f(y)} \mathrm{e}^{i g(y)} \mathrm{d} y
$$

say, with real-valued

$$
f(y)=-y F_{k}^{\prime}(2 T)+\frac{y^{3}}{3 !} F_{k}^{\prime \prime \prime}(T) \ldots, g(y)=-\frac{y^{2}}{2 !} F_{k}^{\prime \prime}(2 T)+\frac{y^{4}}{4 !} F_{k}^{(4)}(2 T)+\ldots
$$

Then we have

$$
\int_{0}^{H}=\int_{0}^{\sqrt{T}}+\int_{\sqrt{T}}^{H}=J_{1}+J_{2}
$$

say. We write $J_{1}$ as

$$
J_{1}=-\frac{1}{F_{k}^{\prime}(2 T)+\frac{y^{2}}{2 !} F_{k}^{\prime \prime \prime}(2 T) \ldots} \int_{0}^{\sqrt{T}} \mathrm{e}^{i g(y)} \mathrm{d}\left(\mathrm{e}^{f(y)}\right)
$$

and integrate by parts. We obtain the same type of exponential integral, only smaller by a factor of

$$
\ll y \frac{F_{k}^{\prime \prime}(2 T)}{F_{k}^{\prime}(2 T)} \ll T^{1 / 2} \cdot \frac{1}{T} \cdot T^{1 / 2-\varepsilon}=T^{-\varepsilon}
$$


since

$$
F_{k}^{\prime}(2 T) \geqslant \log \frac{(T / \pi)^{k / 2}}{n} \geqslant \log \frac{(T / \pi)^{k / 2}}{(T / \pi)^{k / 2}-T^{k / 2-1 / 2+\varepsilon}} \geqslant T^{\varepsilon-1 / 2} .
$$

This means that, after sufficiently many integrations by parts, the ensuing integral will be negligible, while the integrated terms will be $\ll T^{k / 4+\varepsilon}$ as in (4.9). Finally in $J_{2}$

$$
y F_{k}^{\prime}(2 T)-\frac{y^{3}}{3 !} F_{k}^{\prime \prime \prime}(T) \ldots \geqslant C T^{1 / 2} \cdot T^{\varepsilon-1 / 2}=C T^{\varepsilon},
$$

so that $\mathrm{e}^{f(y)}$ is negligibly small. The net result of our considerations is that in the evaluation of the right-hand side of (3.1) the main terms, arising from the saddle point terms, are given by (4.18), while all the error terms are $\ll_{\varepsilon} T^{k / 4+\varepsilon}$.

Remark 4. With a more careful analysis one can get rid of the terms implied by $+\ldots$ in (4.1). The same also follows if one uses an idea of Prof. Matti Jutila, who kindly informed me that the above proof may be simplified as follows. The method may be traced back to E.C. Titchmarsh [28, p. 261], and a sketch is as follows. Note that

$$
\begin{aligned}
& \int_{T}^{2 T} Z^{k}(t) \mathrm{d} t=-i \int_{\frac{1}{2}+i T}^{\frac{1}{2}+2 i T} \chi^{-k / 2}(s) \zeta^{k}(s) \mathrm{d} s \\
& =-i\left(\int_{1+\varepsilon+i T}^{1+\varepsilon+2 i T}+\int_{\frac{1}{2}+i T}^{1+\varepsilon+i T}-\int_{\frac{1}{2}+\varepsilon+i T}^{1+\varepsilon+2 i T}\right) \chi^{-k / 2}(s) \zeta^{k}(s) \mathrm{d} s .
\end{aligned}
$$

On $\sigma=1+\varepsilon$ we have $\zeta^{k}(s)=\sum_{n=1}^{\infty} d_{k}(n) n^{-s}$, so that the above expression is seen to be

$$
\sum_{n=1}^{\infty} d_{k}(n) n^{-1-\varepsilon} \int_{T}^{2 T}\left(\frac{t}{2 \pi}\right)^{\frac{k}{4}+\frac{k \varepsilon}{2}} \mathrm{e}^{i F_{k}(t)} \mathrm{d} t+O_{\varepsilon, k}\left(T^{k / 4+\varepsilon}\right)
$$

for $k \leqslant 4$. The exponential integral is evaluated by (4.17), and Theorem 6 will follow. I am grateful to Prof. Jutila for pointing this out to me.

\section{NATURAL BOUNDARIES}

If a Dirichlet series $F(s)$ has a (meromorphic) continuation to $\Re$ e $s>\sigma_{0}$, then the line $\Re$ e $s=\sigma_{0}$ is said to be the natural boundary of $F(s)$ if the poles of $F(s)$ are dense on $\Re$ e $s=\sigma_{0}$, so that $F(s)$ cannot be continued analytically to $\Re$ e $s \leqslant \sigma_{0}$. The history of natural boundaries for Dirichlet series goes at least back to $\mathrm{T}$. Estermann [9]. For example, one has

$$
\begin{gathered}
\sum_{n=1}^{\infty} d_{k}^{2}(n) n^{-s}=\zeta^{k^{2}}(s) \prod_{p} P_{k}\left(p^{-s}\right) \quad(\Re \mathrm{e} s>1), \\
P_{k}(u):=(1-u)^{2 k-1} \sum_{n=0}^{k}\left(\begin{array}{c}
k-1 \\
n
\end{array}\right)^{2} u^{k},
\end{gathered}
$$


and Estermann showed that the above Euler product has meromorphic continuation to $\Re$ e $s>0$, but has the line $\Re$ e $s=0$ as the natural boundary when $k>2$. In fact, his result holds for a class of Dirichlet series of which the above product is a special case. Estermann's results were generalized by G. Dahlquist [5], and recent investigations include the works of G. Bhowmik and J.-C. Schlage-Puchta $[2],[3]$.

If $A(s)=\sum_{n=1}^{\infty} a_{n} n^{-s}$ in its region of absolute convergence $\Re$ e $s>\sigma_{a}$, then by Perron's inversion formula

$$
\sum_{n \leqslant x} a_{n}=\frac{1}{2 \pi i} \int_{c-i \infty}^{c+i \infty} A(s) \frac{x^{s}}{s} \mathrm{~d} s \quad\left(x \notin \mathbb{N}, c>\sigma_{a}\right) .
$$

In practice one wants to shift the line of integration in (5.1) to the left, to reduce the contribution of the term $x^{s}$. This is possible only if $A(s)$ is holomorphic on the new path. If $\sigma=\sigma_{0}\left(<\sigma_{a}\right)$ is the natural boundary of $F(s)$, then we cannot have $c \leqslant \sigma_{0}$, hence the usefulness of (5.1) is limited if $\sigma_{0}$ exists. This is one of the reasons which makes the study of natural boundaries of Dirichlet series important.

The interest in natural boundaries for $\mathcal{Z}_{k}(s)$ begins with the notes of A. Diaconu [7], followed by the author's notes [16], and the papers of Diaconu, Garrett, Goldfeld [8] and Y. Motohashi [25]. Note that $\mathcal{Z}_{k}(s)$ does not have an Euler product, which makes the problem more difficult. It is conjectured in all these works that the analytic continuation of $\mathcal{Z}_{3}(s)\left(\equiv \mathcal{M}_{6}(s)\right)$ has $\Re$ e $s=\frac{1}{2}$ as the natural boundary, and that, more generally, $\mathcal{Z}_{k}(s)$ for $k \geqslant 3$ has $\Re$ e $s=\frac{1}{2}$ as the natural boundary. A full proof of this important claim concerning $\mathcal{Z}_{k}(s)$ would be most welcome. The basic idea that leads to it is simple, and is open to generalizations. Namely on p. 2 of [6] (or p. 3 of [7]) it is said that the analytic continuation of $(s, w$ are complex variables)

$$
\int_{1}^{\infty}\left(\frac{m}{n}\right)^{i x} L\left(s_{1}+i x\right) L\left(s_{2}-i x\right) x^{-w} \mathrm{~d} x
$$

produces the analytic continuation of

$$
\int_{1}^{\infty}|F(\sigma+i x) L(\sigma+i x)|^{2} x^{-w} \mathrm{~d} x, \quad F(s)=\sum_{n=1}^{\infty} f(n) n^{-s}
$$

under some reasonable conditions, simply by squaring out $|F|^{2}$ and summing over the relevant $m, n$. In Proposition 2.6 on p. 3 this approach is discussed when $L$ is the zeta-function of a holomorphic cusp form of weight $\kappa$ for $S L(2, \mathbb{Z})$.

If in (5.3) we take $F=\zeta, L=\zeta^{2}, \sigma=\frac{1}{2}$, then we have to observe that $\mathcal{Z}_{2}(s)$ has (see Section 1$)$ infinitely many poles at $s=\frac{1}{2} \pm i \kappa_{j}\left(\kappa_{j}=\sqrt{\lambda_{j}-\frac{1}{4}}\right)$. Heuristically, 
when we sum over various $m, n$ in (5.2) to get the analytic continuation of $\mathcal{Z}_{3}(w)$, each of the poles $\frac{1}{2} \pm i \kappa_{j}$ will be somewhat perturbed. Their totality will be dense on the $\frac{1}{2}$-line, and will produce the $\frac{1}{2}$-line as the natural boundary for $\mathcal{Z}_{3}(w)$. Inasmuch as this seems plausible, a rigorous proof is in order.

Suppose that one has found the analytic continuation of $\mathcal{Z}_{3}(w)$ to the right of the $\frac{1}{2}$-line. Then it is seems plausible that $\mathcal{Z}_{3}(w)$ (being more complex that $\left.\mathcal{Z}_{2}(w)\right)$ will have infinitely many poles as well. Where are these poles located? One does not expect them be too near the $\frac{1}{2}$-line, so the $\frac{3}{4}$-line is a very good candidate to contain infinitely many poles of $\mathcal{Z}_{3}(w)$. But by the principle inherent in $(5.2)-(5.3)$, then the $\frac{3}{4}$-line would be a natural barrier for $\mathcal{Z}_{4}(w)$, and so on each $\mathcal{Z}_{k}(w)$ would, with increasing $k$, have poles nearing the 1 -line.

The recent work of Y. Motohashi [25] on (5.3) (when $L=\zeta^{2}$ ) supports the claim that $\mathcal{Z}_{3}(s)$ has $\sigma=1 / 2$ as the natural boundary. The author says: "Our theorem suggests that the Mellin transform $\int_{1}^{\infty}\left|\zeta\left(\frac{1}{2}+i x\right)\right|^{6} x^{-s} \mathrm{~d} x$ should have the line $\Re$ e $s=1 / 2$ as a natural boundary... The same was also speculated also by a few people other than us, but it appears that our theorem is so far the sole explicit evidence supporting the observation."

The natural boundary of $\mathcal{Z}_{3}(s)$ on $\Re$ e $s=1 / 2$ indicates certainly a complicated structure of the error term $E_{3}(T)$ for the sixth moment of $\left|\zeta\left(\frac{1}{2}+i t\right)\right|$, but in itself it does not exclude the possibility of the bound $E_{3}(T) \ll_{\varepsilon} T^{1 / 2+\varepsilon}$. If $E_{3}(T) \ll_{\varepsilon} T^{\theta+\varepsilon}$ with $\theta$ as small as possible, then $\mathcal{Z}_{3}(s)$ would have singularities on $\Re$ e $s=\theta$, if $1 / 2<\theta<1$. Inasmuch as it seems plausible (to me) that $\theta=3 / 4$, this is a major unsolved problem.

Remark 5. I believe that $\left(P_{9}(y)\right.$ is an explicit polynomial of degree nine)

$$
\begin{aligned}
& \int_{0}^{T}\left|\zeta\left(\frac{1}{2}+i t\right)\right|^{6} \mathrm{~d} t=T P_{9}(\log T)+E_{3}(T), \\
& E_{3}(T)=O_{\varepsilon}\left(T^{3 / 4+\varepsilon}\right), \quad E_{3}(T)=\Omega\left(T^{3 / 4}\right)
\end{aligned}
$$

holds, where the main term $T P_{9}(\log T)$ is the one predicted by Conrey et al. [4]. However in [4] the error term is indicated to be (in all cases) $O_{\varepsilon}\left(T^{1 / 2+\varepsilon}\right)$, which I do not think can be true.

In what concerns the true order of higher moments of $\left|\zeta\left(\frac{1}{2}+i t\right)\right|$, the situation is even more unclear. Already for the eighth moment it is hard to ascertain what goes on, much less for the higher moments. The main term for the general $2 k$-th moment should involve a main term of the type suggested by [2], but it could turn out that the error term

$$
E_{k}(T)=\int_{0}^{T}\left|\zeta\left(\frac{1}{2}+i t\right)\right|^{2 k} \mathrm{~d} t-T P_{k^{2}}(\log T) \quad(k \in \mathbb{N})
$$

in the general case (when $k \geqslant 4$ ) contains expressions which make it larger than the term $T P_{k^{2}}(\log T)$. For this see the discussion in [12] (also [24, pp. 218-219]). 
Essentially the argument is as follows. In general, from the knowledge about the order of $E_{k}(T)$ one can deduce a bound for $\zeta\left(\frac{1}{2}+i T\right)$ via the estimate

$$
\zeta\left(\frac{1}{2}+i T\right) \ll(\log T)^{\left(k^{2}+1\right) /(2 k)}+\left(\log T \max _{t \in[T-1, T+1]}\left|E_{k}(t)\right|\right)^{1 /(2 k)},
$$

which is Lemma 4.2 of [11]. The conjectured bounds

$$
E_{k}(T) \ll_{\varepsilon} T^{k / 4+\varepsilon} \quad(k \leqslant 4)
$$

all imply $\zeta\left(\frac{1}{2}+i t\right) \ll_{\varepsilon}|t|^{1 / 8+\varepsilon}$, which is out of reach at present, but is still much weaker than the Lindelöf hypothesis that $\zeta\left(\frac{1}{2}+i t\right) \ll_{\varepsilon}|t|^{\varepsilon}$. On the other hand, we know that the omega-result

$$
E_{k}(T)=\Omega\left(T^{k / 4}\right)
$$

hold for $k=1,2$, and as already explained, there are reasons to believe that (5.7) holds for $k=3$. Perhaps it holds for $k=4$ also, but the truth of (5.7) for any $k>4$ would imply that the Lindelöf hypothesis is false, and ipse facto the falsity of the Riemann hypothesis (that all complex zeros of $\zeta(s)$ satisfy $\Re$ e $s=1 / 2$ ). Namely it is well-known (see e.g., [10] or [28]) that the Riemann hypothesis implies even $\log \left|\zeta\left(\frac{1}{2}+i t\right)\right| \ll \log |t| / \log \log |t|$, which is stronger than the Lindelöf hypothesis $\left(\Leftrightarrow \log \left|\zeta\left(\frac{1}{2}+i t\right)\right| \ll_{\varepsilon} \varepsilon \log |t|\right)$. The reason why, in general, (5.7) makes sense is that a bound $E_{k}(T) \ll T^{c_{k}}$ for some fixed $k(>4)$ with $c_{k}<k / 4$ would imply (by (5.5)) the bound $\zeta\left(\frac{1}{2}+i t\right) \ll_{\varepsilon}|t|^{c_{k} /(2 k)+\varepsilon}$ with $c_{k} /(2 k)<1 / 8$. But the most one can get (by using (5.5)) from the error term in the mean square and the fourth moment of $\left|\zeta\left(\frac{1}{2}+i t\right)\right|$ is the bound

$$
\zeta\left(\frac{1}{2}+i t\right) \ll_{\varepsilon}|t|^{1 / 8+\varepsilon} .
$$

It does not appear likely to me that, say from the twelfth moment $(k=6)$, one will get a better pointwise estimate for $\zeta\left(\frac{1}{2}+i t\right)$ than what one can get from the mean square formula $(k=1)$. Nothing, of course, precludes yet that this does not happen, just that it appears to me not to be likely. As in all such dilemmas, only rigorous proofs will reveal in due time the real truth.

\section{REFERENCES}

[1] F.V. Atkinson, The mean value of the Riemann zeta-function, Acta Math. 81 (1949), 353-376.

[2] G. Bhowmik and J.-C. Schlage-Puchta, Natural boundaries of Dirichlet series, Func. Approx. Comment. Math. 37(2007), 17-29.

[3] G. Bhowmik and J.-C. Schlage-Puchta, Essential singularities of Euler products, to appear, see arXiv:1001.1891.

[4] J.B. Conrey, D.W. Farmer, J.P. Keating, M.O. Rubinstein and N.C. Snaith, Integral moments of $L$-functions, Proc. London Math. Soc. (3) 91(2005), 33-104. 
[5] G. Dahlquist, On the analytic continuation of Eulerian products, Ark Mat. 1(1952), 533-554.

[6] A. Diaconu, The function $\mathcal{Z}_{3}(w)$ has natural boundary, Notes of October 24, 2006.

[7] A. Diaconu, P. Garrett and D. Goldfeld, Natural boundaries and a correct notion of integral moments of $L$-functions, preprint, 2009.

[8] A. Diaconu, D. Goldfeld and J. Hoffstein, Multiple Dirichlet series and moments of zeta and $L$-functions Compos. Math. 139(2003), 297-360.

[9] T. Estermann, On certain functions represented by Dirichlet series, Proc. London Math. Soc. $\mathbf{2 7}(1926)$, 435-448.

[10] A. Ivić, The Riemann zeta-function, John Wiley \& Sons, New York 1985 (2nd edition. Dover, Mineola, New York, 2003).

[11] A. Ivić, The mean values of the Riemann zeta-function, LNs 82, Tata Inst. of Fundamental Research, Bombay 1991 (distr. by Springer Verlag, Berlin etc.).

[12] A. Ivić, On some conjectures and results for the Riemann zeta-function and Hecke series, Acta Arithmetica 109(2001), 115-145.

[13] A. Ivić, On the integral of Hardy's function, Arch. Mathematik 83(2004), 41-47.

[14] A. Ivić, The Mellin transform of the square of Riemann's zeta-function, International J. of Number Theory 1(2005), 65-73.

[15] A. Ivić, The Laplace and Mellin transforms of powers of the Riemann zeta-function, International Journal of Mathematics and Analysis 1(2), 2006, 113-140.

[16] A. Ivić, Remarks on the natural boundary of $\mathcal{Z}_{k}(s)$, notes of October 2006 and October 2009.

[17] A. Ivić, On some reasons for doubting the Riemann Hypothesis, in "The Riemann Hypothesis", P. Borwein et al., CMS Books in Mathematics, Springer, 2008, pp. 131-160.

[18] A. Ivić, M. Jutila and Y. Motohashi, The Mellin transform of powers of the zeta-function, Acta Arithmetica 95(2000), 305-342.

[19] M. Jutila, The Mellin transform of the square of Riemann's zeta-function, Periodica Math. Hungarica 42(2001), 179-190.

[20] M. Jutila, Atkinson's formula for Hardy's function, J. Number Theory 129(2009), 2853-2878.

[21] A.A. Karatsuba and S.M. Voronin, The Riemann zeta-function, Walter de Gruyter, Berlin etc., 1992.

[22] M.A. Korolev, On the integral of Hardy's function $Z(t)$, Izv. Math. 72, No. 3, 429-478 (2008); translation from Izv. Ross. Akad. Nauk, Ser. Mat. 72, No. 3, 19-68 (2008).

[23] M. Lukkarinen, The Mellin transform of the square of Riemann's zeta-function and Atkinson's formula, Ann. Acad. Sci. Fenn. Math. Diss. 140, 2007.

[24] Y. Motohashi, Spectral theory of the Riemann zeta-function, Cambridge University Press, Cambridge, 1997.

[25] Y. Motohashi, The Riemann zeta-function and Hecke congruence subgroups II, Journal of Research Institute of Science and Technology, Tokyo, 2009, to appear.

[26] K. Ramachandra, On the mean-value and omega-theorems for the Riemann zeta-function, Tata Inst. of Fundamental Research, (distr. by Springer Verlag, Berlin etc.), Bombay, 1995.

[27] E.C. Titchmarsh, Introduction to the Theory of Fourier Integrals, Clarendon Press, Oxford, 1948.

[28] E.C. Titchmarsh, The theory of the Riemann zeta-function (2nd edition), University Press, Oxford, 1986.

Katedra Matematike RGF-A, Universitet u Beogradu, Đušina 7, 11000 Beograd, SERBIA.

E-mail address: ivic@rgf.bg.ac.rs, aivic@matf.bg.ac.rs 